\title{
COMPARATIVO ENTRE OS TEMPOS DE VISUALIZAÇÃO PARA OPERAR DISPOSITIVOS COM INTERFACES FÍSICAS E DISPOSITIVOS COM DISPLAYS SENSÍVEIS AO TOQUE EM AUTOMÓVEIS
}

\author{
Rafael Silva Duarte \\ Universidade Federal do Rio Grande do Sul - UFRGS \\ raffael23@gmail.com \\ Branca Freitas de Oliveira \\ Universidade Federal do Rio Grande do Sul - UFRGS \\ branca@ufrgs.br
}

Resumo: Este trabalho trata da comparação dos tempos de visualização do usuário para executar uma tarefa secundária, no caso, operar um rádio automotivo, em diferentes interfaces, enquanto dirige um automóvel em uma simulação.

Para os testes, realizados com 21 pessoas, entre 18 e 72 anos, foi construído um simulador utilizando um videogame e acessórios como volante, pedais e câmbio. Para as diferentes interfaces nos testes foram utilizados um rádio automotivo convencional, uma cópia virtual touchscreen desse e mais uma segunda versão virtual, também sensível ao toque, mas com layout diferenciado.

Enquanto testavam cada uma das versões de interface em situações semelhantes no simulador, os participantes eram filmados, e o vídeo era posteriormente analisado para determinar o tempo que cada usuário desviava o olhar do monitor principal para realizar a tarefa requisitada.

Os resultados obtidos foram comparados a fim de determinar o impacto que a substituição de dispositivos convencionais por aparelhos com tecnologias touchscreen têm na atenção dos motoristas.

Palavras-chave: Tecnologias touchscreen, interfaces automotivas, tempo de visualização.

\footnotetext{
Abstract: This paper compares user's glance times to execute a secondary task, in this case, to operate a car radio, in different interfaces, while driving an automobile in a simulation.

For the tests, conducted with 21 participants, between 18 and 72 years old, a simulator was built using a videogame equipped with steering wheel, pedals and shift gears. For the different interfaces, the tests used a conventional car radio, a virtual touchscreen copy of this radio, and a modified touchscreen interface.
} 
While every interface were tested in similar simulated conditions, the participants were filmed, and the video was analyzed to determine the time that each user look away from the main monitor to realized the required task.

The results were compared to determine the impact of replacing conventional devices for touchscreen technologies in the driver's attention.

Keywords: Touchscreen technologies, automotive interfaces, glance time.

\section{INTRODUÇÃO}

A tecnologia touchscreen permite que usuários utilizem a tela de um dispositivo como um painel de comando, usando-a para inserir dados no produto. 0 sistema de toque é um design de interface entre o produto e o usuário, permitindo que o consumidor comande esse produto de forma intuitiva, interagindo com os elementos conforme eles aparecem na tela. A invenção da tecnologia é creditada a Sam Hurst, que desenvolveu uma tela para digitalização de dados chamada de Elograph, enquanto trabalhava na Universidade de Kentucky, Estados Unidos.

Porém, apesar de já ter sido empregada em alguns dispositivos anteriormente, a tecnologia apenas se difundiu entre o público após o lançamento do iPhone, pela Apple. O smartphone foi comercializado em junho de 2007 e, até o final de 2010, havia vendido 90 milhões de unidade, respondendo por metade dos lucros do ramo de aparelhos de telefonia móvel.

O sucesso comercial do iPhone e do Ipad, posteriormente, mudou o rumo das indústrias. Todas as empresas de comunicação aderiram à interface sensível ao toque e à conectividade que esses dispositivos proporcionaram aos consumidores. Eles mudaram não apenas o modo como as pessoas se relacionam e como percebem o mundo a sua volta, mas também o que elas esperam de um produto, em termos de facilidade de operação.

Nós estamos vivendo a Era dos dispositivos all-in-one conectados - a Era da consolidação dos hardwares. O iPhone trouxe essa Era, em que fabricantes de hardwares estão competindo com plataformas que prometem oferecer qualquer coisa, a qualquer hora, em qualquer lugar, tudo com o apertar de um botão. (CHEN, 2011, p.39)

$\mathrm{E}$, embora alguns automóveis modernos ofereçam tal tecnologia, como o novo Porsche 918, com seu console touchscreen, a maioria da indústria automotiva ainda converge lentamente para essa nova realidade, às vezes oferecendo a tecnologia de forma mesclada com a antiga, de botões físicos. Em parte, a razão de tal lentidão para incorporar a tecnologia sensível ao toque está nas consequências em termos de segurança, já que, impossibilitado de fazer a operação às cegas, mais atenção é requerida do usuário para operar o dispositivo.

Nesse sentido, o objetivo principal desse trabalho é medir o tempo de visualização necessário para acionar comandos em interfaces físicas e sensíveis ao toque.

Os objetivos secundários são:

- Medir o tempo de desvio de olhar da tarefa principal enquanto os usuários realizam uma tarefa secundária num dispositivo com interface física; 
- Medir o tempo de desvio de olhar nas mesmas condições, enquanto o usuário utiliza uma cópia virtual sensível ao toque da interface física;

- $\quad$ Averiguar como a modificação da interface virtual, utilizando comandos mais visíveis, afeta os resultados obtidos com as demais interfaces testadas.

\section{METODOLOGIA}

A parte física do experimento consiste em um simulador de automóvel (Figura 1), montado a partir de um videogame Sony Playstation 3, conectado a um monitor LCD de 26 polegadas e um volante $G 25$, equipado com três pedais e transmissão manual. O aparelho roda o jogo Gran Turismo 6, em condições pré-determinadas. Próximo ao câmbio, no lado direito do simulador, é montado um rádio automotivo Pioneer DEH-P3580MP ou um iPad, dependendo do teste. Uma câmera Nikon D7000 fica montada atrás do monitor, voltada para o usuário do simulador.

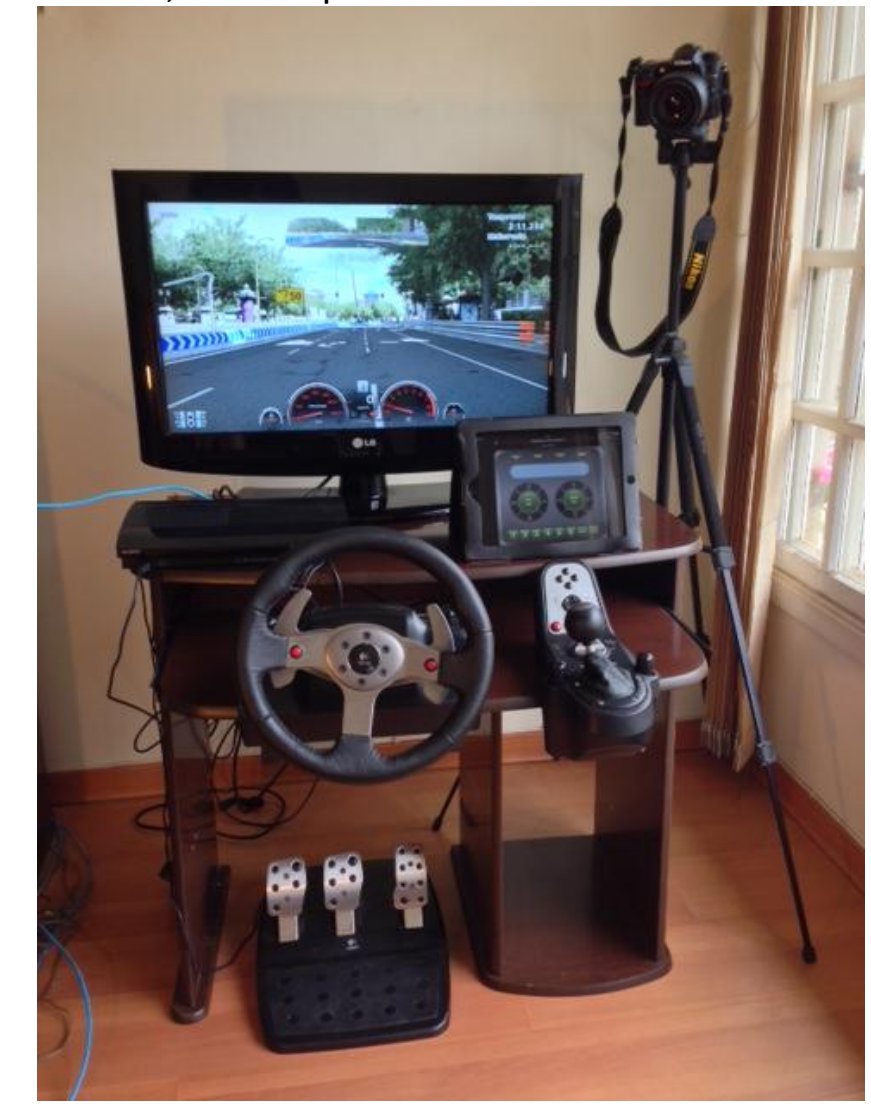

Figura 1 - Simulador de automóvel

Fonte: Elaborado pelo autor

Um percurso de três minutos é traçado no simulador e, enquanto o usuário percorre o trajeto, ele deve, conforme orientado, realizar as operações de ligar o rádio, trocar de estação e aumentar o volume, nessa ordem. Cada usuário faz o mesmo percurso três vezes: a primeira operando o rádio com interface física, a segunda operando a versão touchscreen do mesmo aparelho via iPad, e a terceira operando uma versão sensível ao toque com interface modificada, conforme a área de display disponível no tablet.

Enquanto o usuário realiza a tarefa, é filmado pela câmera, em resolução de 1080 pixels. Posteriormente, os vídeos são analisados quadro a quadro para medir o tempo que o olho desviou do monitor principal para realizar a tarefa requisitada. 


\subsection{Participantes e treinamento}

O grupo analisado consistiu-se de 21 pessoas, separados da seguinte forma: 4 pessoas na faixa etária de 18 a 24 anos; 8 pessoas na faixa dos 25 aos 39 anos; 5 pessoas na faixa dos 40 aos 54 anos; 4 pessoas com 55 anos ou mais.

Antes dos testes, um pequeno questionário foi aplicado aos participantes, questionando-os acerca de suas experiências como motoristas e familiaridade com dispositivos sensíveis ao toque. Apenas dois participantes não eram habilitados e todos revelaram ter pelo menos um dispositivo touchscreen de uso frequente.

Também, antes de serem testados, foi permitido que os usuários conhecessem o simulador, dirigindo o mesmo percurso, nas mesmas condições, até que se sentissem aptos a realizar o percurso. Os usuários também foram apresentados as três interfaces com as quais iriam interagir, permitindo familiaridade com a operação dos mesmos.

Após os testes, os participantes eram novamente questionados sobre suas impressões em relação às diferentes interfaces que foram utilizadas nos testes.

\subsection{Interfaces utilizadas}

No primeiro teste, os usuários utilizam a interface $A$, um rádio automotivo comum, modelo Pioneer DEH-P3580MP (Figura 2), com feedback tátil, já que os botões físicos podem ser sentidos, e há uma confirmação auditiva, através de um "bip" quando um botão é pressionado.

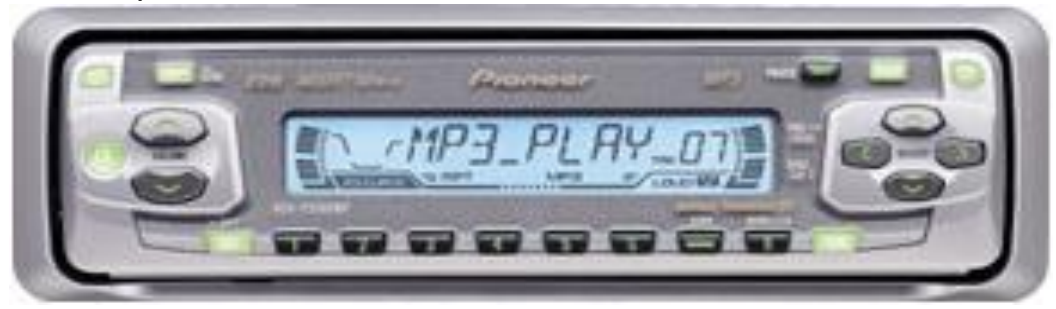

Figura 2 - Interface A - Rádio automotivo comum

Fonte: Elaborado pelo autor

O segundo teste é realizado com uma cópia virtual idêntica do modelo Pioneer DEH-P3580MP, tanto em layout, quanto em cores e dimensão (Figura 3), sendo chamado de interface B. O modelo para ser utilizado no primeiro teste touchscreen foi desenvolvido através dos softwares Adobe Illustrator e Adobe Photoshop e foi carregado em uma página da internet. Assim, quando o usuário pressiona uma tecla, um feedback auditivo semelhante ao do modelo real é gerado. O modelo virtual é então carregado no iPad, que substitui o rádio real, e o teste é repetido. $\mathrm{O}$ resultado entre dois modelos idênticos, um real e um touchscreen, deve ilustrar, para os fins desse estudo, a diferença do tempo de visualização entre as duas tecnologias.

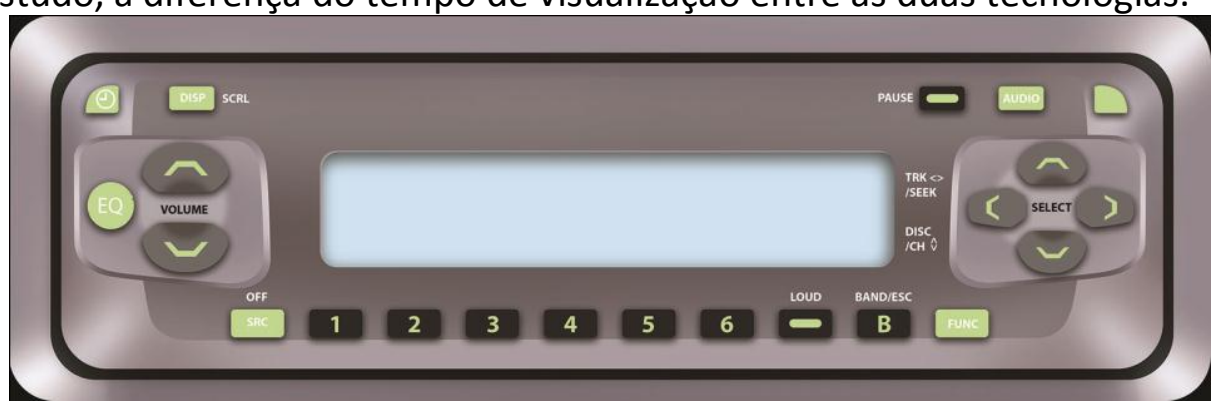

Figura 3 - Interface B - Cópia virtual do rádio automotivo Fonte: Elaborado pelo autor 
Para o terceiro e último teste, uma versão sensível ao toque foi desenvolvida, utilizando botões maiores e mais visíveis, sendo chamado de interface C (figura 4). Embora o layout tenha sido modificado, o esquema de cores e o número de comandos permaneceram inalterados. A intenção desse modelo é determinar como o melhor aproveitamento da tecnologia touchscreen influencia o tempo de visualização constatado nos testes com a interface B.

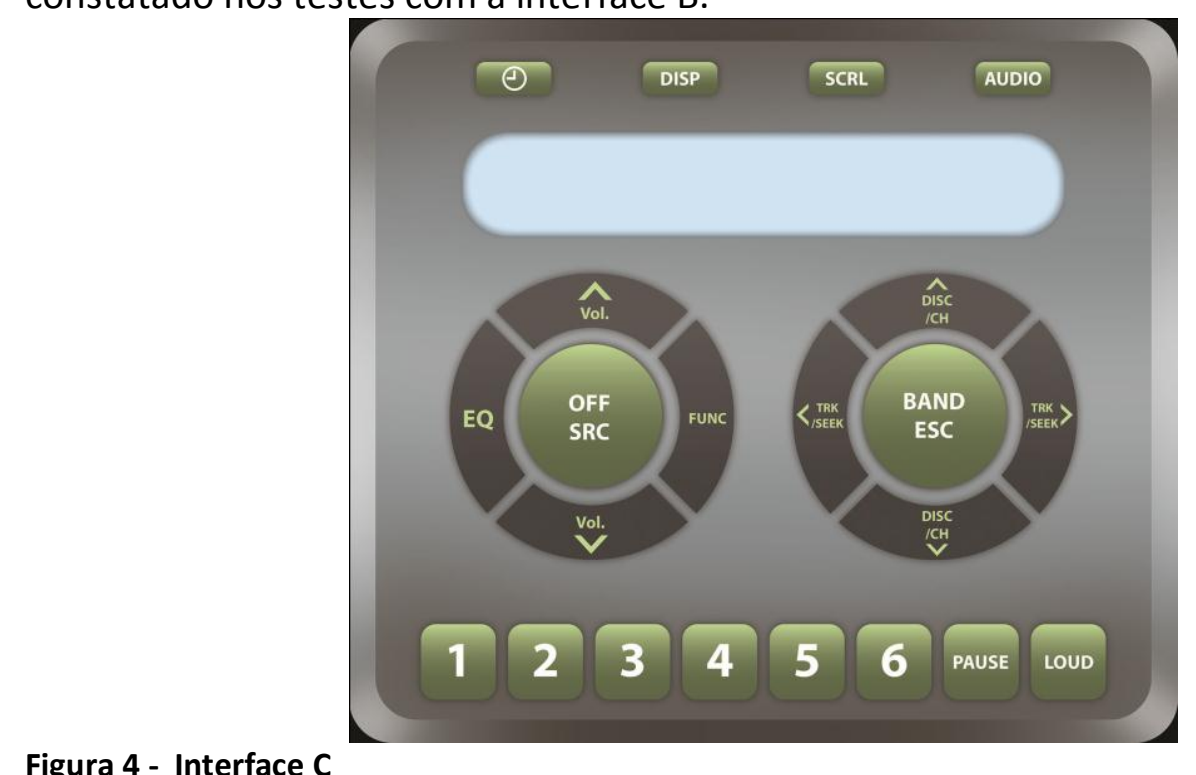

Figura 4 - Interface C

Fonte: Elaborado pelo autor

\section{RESULTADOS}

Como cada uma das tarefas realizadas (ligar o rádio, trocar de estação e aumentar o volume) é realizada através de botões de diferentes dimensões e localizações, a fim de padronizar os resultados, os tempos referentes as três tarefas foram somados e divididos pelo número de operações, obtendo-se, assim, o tempo médio de visualização que cada usuário utiliza para cumprir uma tarefa qualquer, ao operar o desportivo em questão.

\subsection{Resultados utilizando a interface $A$}

A primeira rodada de testes foi realizada com o intuito de se obter uma medição de referência para comparar com as outras interfaces. Nesse teste, realizado com a interface A (rádio automotivo Pioneer modelo DEH-P3580MP a média dos tempos de visualização medidos para realizar as tarefas requisitadas foi de 1,79 segundo para os usuários situados na faixa dos 18 aos 24 anos, de 1,81 segundo para o grupo de 25 a 39 anos, 1,91 segundo para o grupo de usuários situados entre 40 e 54 anos e, entre o grupo dos usuários com 55 anos ou mais, a média subiu para 1,99 segundo. Os resultados dos testes com a primeira interface podem ser verificado no gráfico 1. 
Gráfico 1 - Média dos tempos na Interface A

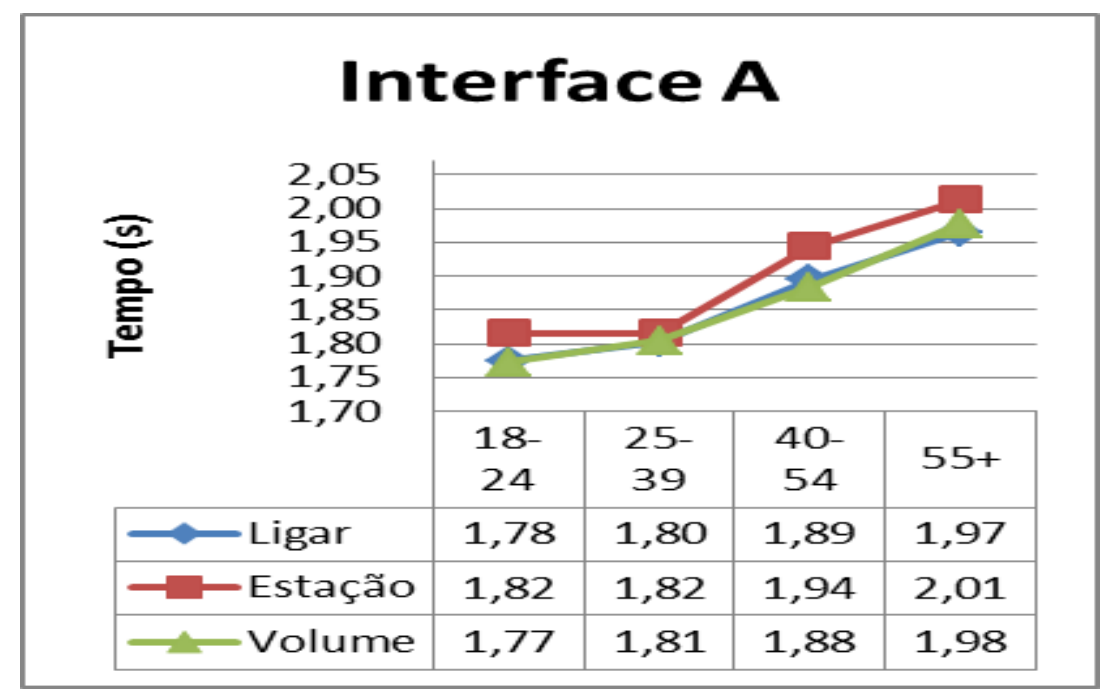

Fonte: Elaborado pelo autor

\subsection{Resultados utilizando a interface $B$}

Na segunda rodada de testes, o dispositivo de interface física foi substituído pelo iPad, apresentando uma cópia virtual sensível ao toque do primeiro equipamento, a fim de comparar os resultados de ambas as tecnologias em layouts idênticos. Os resultados obtidos, conforme mostrado no gráfico 2, foram de 1,82 segundo para 0 grupo de 18 a 24 anos, de 1,85 segundo para o grupo de usuários dos 25 aos 39 anos, de 1,96 segundo para o grupo de 40 a 54 anos e de 2,05 segundos para usuários com 55 anos ou mais.

Gráfico 2- Média de tempos na Interface B

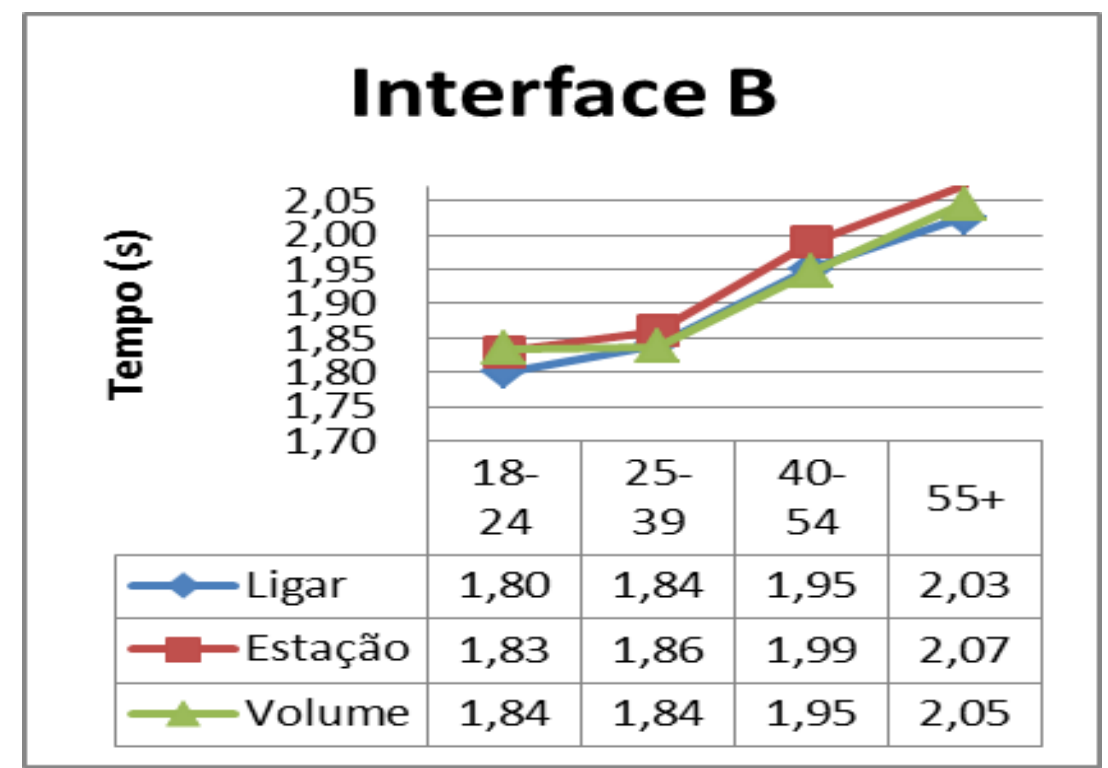

Fonte: Elaborado pelo autor

\subsection{Resultados utilizando a interface $C$}

Para o terceiro teste, a interface $B$ foi modificada, redimensionando e alterando a posição de botões no layout. Nesse teste, conforme apresentado no gráfico 3 , os 
tempos medidos foram de 1,80 segundo para o grupo de usuários dos 18 aos 24 anos; 1,82 para o grupo de pessoas entre 25 e 39 anos; 1,93 para o grupo com idades entre 40 e 54 anos; e o grupo com 55 anos ou mais, apresentou uma média de tempo de 1,98 segundo.

Gráfico 3 - Média dos tempos na Interface C

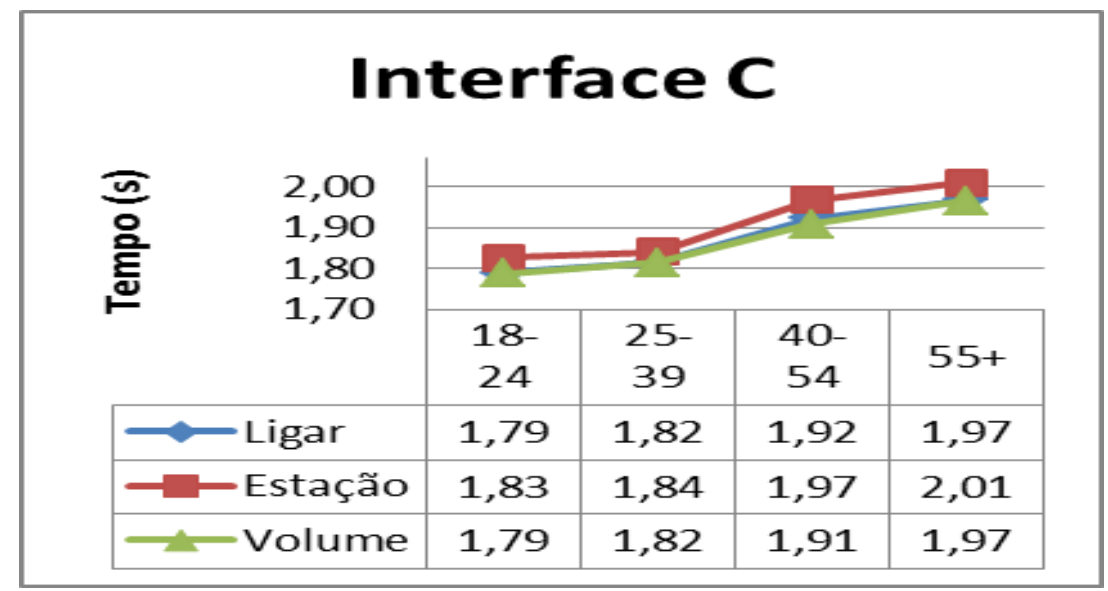

Fonte: Elaborado pelo autor

\subsection{Análise dos resultados}

A primeira constatação dos resultados, conforme pode ser verificado no gráfico 4, é que o tempo médio de visualização ao realizar as tarefas aumenta conforme aumenta a idade do grupo testado, em todas as interfaces.

Gráfico 4 - Comparação dos tempos médios nas Interfaces A, B e C

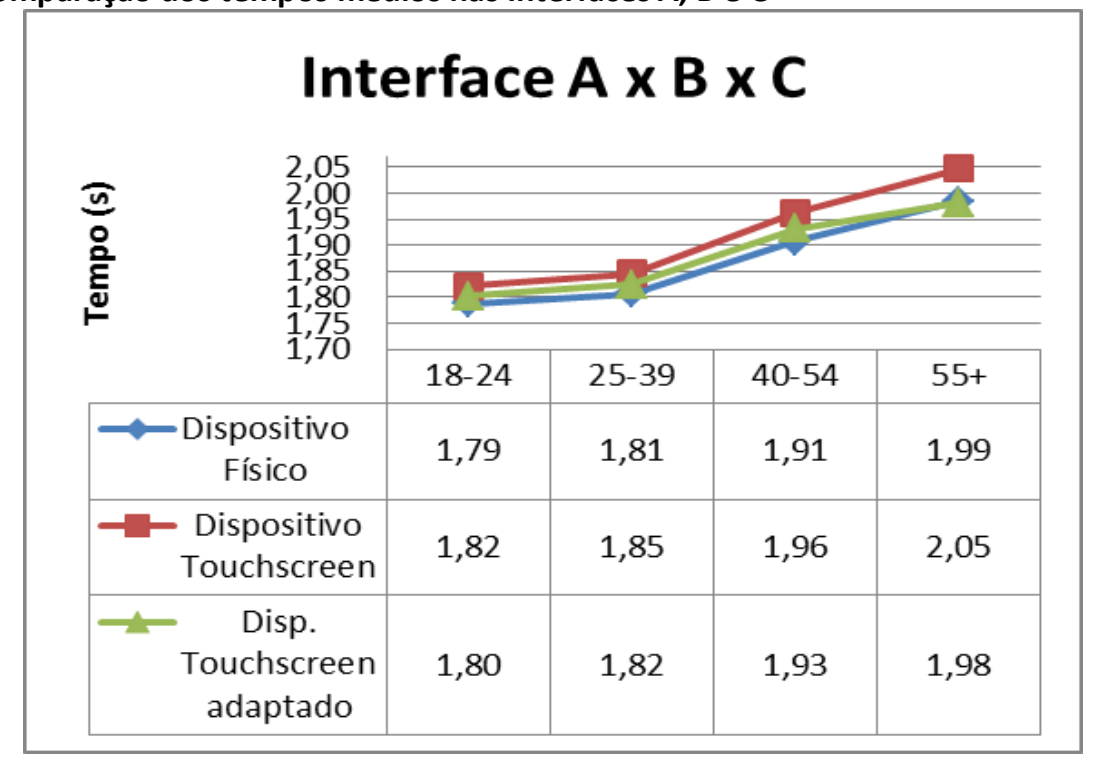

Fonte: Elaborado pelo autor

Comparando os resultados das interfaces A e B constata-se um aumento do tempo de visualização para todos os grupos e idade, ao trocar o dispositivo físico pelo virtual. Porém, a diferença nos tempos medidos é menor nos dois grupos mais jovens do que nos dois grupos com idade mais avançada.

Analisando os dados da pesquisa realizada com os participantes, percebe-se que os mais jovens possuem mais dispositivos com interface touchscreen do que os 
mais velhos, além de os utilizarem por períodos maiores durante o dia. Logo, a menor diferença do tempo de visualização do dispositivo sensível ao toque em relação ao físico, entre os grupos mais jovens, pode ser creditada a uma maior familiaridade desses grupos com as novas tecnologias.

Porém, quando a interface B é comparada com a C, os tempos de visualização de todos os grupos diminuem na versão modificada, embora ainda fiquem acima dos tempos aferidos com a interface $A$ em três dos quatro grupos testados. A exceção cabe ao grupo mais velho, com pessoas de 55 anos ou mais, cuja média de tempo baixou de 1,99 segundo durante o teste de referência, com dispositivo físico, para 1,98 segundo com a interface $\mathrm{C}$.

Conforme os dados do questionário aplicado após os testes, três dos quatro integrantes do grupo mais velho alegou preferir a interface $C$, por causa dos botões mais visíveis. A interface $C$ também foi a preferida de outros 6 participantes dos testes, sendo a escolha de 9 dos 21 usuários, que alegaram, além da facilidade de visualização, a melhor organização do layout como fator determinante para sua escolha. Em seguida, a interface $A$ foi a preferida de 8 participantes, que alegaram, em sua quase totalidade, que o elemento tátil a tornava a melhor opção. $A$ interface $B$ ficou em último lugar, sendo a preferência de apenas 4 participantes.

\subsection{Oportunidade de Melhoramento}

A pequena diferença dos tempos medidos, e a polarização na preferência dos participantes entre os modelos $\mathrm{A}$ e $\mathrm{C}$, evidenciam que os consumidores preferem os layouts mais inteligentes, gerados a partir de interfaces virtuais sensíveis ao toque, porém, gostariam de também contar com 0 elemento tátil dos produtos convencionais.

Novas tecnologias de displays touchscreen com feedback tátil estão em fase final de desenvolvimento e, quando lançados no mercado, podem ser o produto ideal para aplicação em dispositivos automotivos, por unirem as possibilidades de customização dos modernos aparelhos touchscreeen à facilidade de manuseio dos dispositivos convencionais.

Um teste utilizando um display sensível ao toque com feedback tátil completaria o estudo, possivelmente apresentando números inferiores aos medidos em todas as interfaces

\section{CONSIDERAÇÕES FINAIS}

Vivemos uma época de mudanças. A adoção de tecnologias sensíveis ao toque é inevitável, em todos os setores de produtos industrializados. Existe, conforme averiguado nesse trabalho, um aumento no tempo de visualização ao mudar de interfaces com botões físicos para displays touchscreen. Porém, Interfaces sensíveis ao toque podem ser modificadas de acordo com as necessidades dos usuários, se adaptando ergonomicamente a todos os tipos de pessoas e agradando a todos os gostos e preferências.

Foi demonstrado neste trabalho que, apesar dos resultados gerais inferiores de tecnologias sensíveis ao toque em relação à dispositivos com botões físicos, em determinadas circunstâncias, mesmo uma interface touchscreen simples, sem feedback tátil, pode apresentar uma resposta melhor do que a tecnologia tradicional. 
Os novos avanços na área de tecnologia touchscreen, principalmente no sentido de possibilitar feedback tátil ao usuário, podem fazer com que a indústria automotiva adote a tecnologia em larga escala, ao aliar possibilidade de personalização com segurança.

\section{REFERÊNCIAS}

CHEN, Brian X. Always On. How the iPhone Unlocked the Anything-AnytimeAnywhere Future - ad Locked Us in. Philadelphia, Pa: Da Capo Press, 2011.

MONE, Gregory. The Future is Flexible Displays. Communications of the ACM Magazine.Volume 56 Issue 6, Jun/2013. p.16-17. Disponível em http://dl.acm.org.ez45. periodicos.capes.gov.br/citation.cfm?doid=2461256.2461263\& CFID $=370033180 \&$ CFTOKEN=23203678 Acessado em 13/10/213.

OHNSMAN, Alan. FIXMER, Andy. ICar Dream Downsizes to Dashboards as Apple Takes on Foes. Disponível em: http://www.bloomberg.com/news/2013-07-30/icar-dreamdownsizes-to-dashboards-as-apple-takes-on-foes.html Acessado em 16/10/2013.

PITTS, Matthew J. et al. Visual-haptic feedback interaction in automotive touchscreens.Displays. Disponível em: www.sciencedirect.com/science/article/pii/S0141938211000874 Acessado em $13 / 10 / 2013$.

PREECE, Jennifer; ROGERS, Yvonne; SHARP, Helen. Design de Interação: além da interação homem-computador. Bookman. Porto Alegre: 2005.

SHNEIDERMAN, Ben; PLAISANT, Catherine.Designing the User Interface - Strategies for Effective Human-Computer Interaction.Fourth Edition. Pearson Education, Inc. 2005. 